\title{
LHC search for di-Higgs decays of stoponium and other scalars in events with two photons and two bottom jets
}

\author{
Nilanjana Kumar ${ }^{1}$ and Stephen P. Martin ${ }^{1,2}$ \\ ${ }^{1}$ Department of Physics, Northern Illinois University, DeKalb IL 60115 \\ ${ }^{2}$ Fermi National Accelerator Laboratory, P.O. Box 500, Batavia IL 60510
}

\begin{abstract}
We study the prospects for LHC discovery of a narrow resonance that decays to two Higgs bosons, using the final state of two photons and two bottom jets. Our work is motivated in part by a scenario in which two-body flavor-preserving decays of the top squark are kinematically forbidden. Stoponium, a hadronic bound state of the top squark and its anti-particle, will then form, and may have a large branching fraction into the two Higgs boson final state. We estimate the cross-section needed for a 5-sigma discovery at the $14 \mathrm{TeV}$ LHC for such a narrow di-Higgs resonance, using the invariant mass distributions of the final state bottom jets and photons, as a function of the integrated luminosity. The results are also applicable to any other di-Higgs resonance produced by gluon fusion.
\end{abstract}

\section{Contents}

\section{Introduction}

II. Event generation and simulation

III. Event selection

References

\section{INTRODUCTION}

ATLAS [1, 2] and CMS [3, 4] have confirmed the existence of a resonance with properties that are consistent with a minimal Standard Model Higgs scalar boson, $h$, with a mass near $126 \mathrm{GeV}$. The precise value of $m_{h}$ is already known at roughly the $1 \%$ level, and will surely improve in the future. This provides an opportunity to search for new physics that lies beyond the Standard Model, by looking for new heavy particles that decay into $h$, exploiting the Higgs boson as a standard candle. 
One such possibility is stoponium, $\eta_{\tilde{t}}$, a bound state of a top squark (stop) and its antiparticle. The stop will be stable enough to hadronize provided that it has no flavor-preserving two-body decays. The binding energy of the $J^{P C}=0^{++}$ground state of stoponium is of order a few $\mathrm{GeV}$, and its width is typically about two orders of magnitude smaller. It will decay primarily by annihilation into pairs of Standard Model particles, including final states $g g, W W, Z Z, h h, \gamma \gamma, Z \gamma, t \bar{t}$, and $b \bar{b}$, as well as pairs of neutralinos, depending on the masses and the stop mixing angle and other supersymmetry-breaking parameters [5] 7 ]. Therefore one can search for narrow invariant mass peaks of stoponium at the LHC or at future hadron colliders. The diphoton final state, as originally proposed in [5, 6] and studied more recently in [7 9] is a promising one due to its clean experimental signature and the excellent diphoton mass resolution of the LHC detectors. The $Z Z$ and $W W$ final states may also provide a viable discovery signature [10, 11]. Early work on stoponium at hadron colliders can be found in [12 15], and discussions of stoponium at linear colliders have been presented in [16 18].

If the stop mass is at least a few $\mathrm{GeV}$ larger than $m_{h}$, then the decay $\eta_{\tilde{t}} \rightarrow h h$ is kinematically allowed and also potentially observable [14], and can easily have a branching ratio of tens of per cent. This possibility was explored in early work for the case $2 m_{h}<$ $m_{\eta_{\tilde{t}}}<m_{W}$ in ref. [14]. In some more modern models, this decay can even have the dominant branching ratio if $m_{n_{\tilde{r}}}$ is not too far above the threshold $2 m_{h}$; see for example the model lines in Figure 8 in ref. [7], which illustrate cases with $\operatorname{BR}\left(\eta_{\tilde{t}} \rightarrow h h\right)>0.7$. The $\operatorname{BR}\left(\eta_{\tilde{t}} \rightarrow h h\right)$ tends to decrease slowly as $m_{\eta_{\tilde{t}}}$ moves far above threshold. The combination of the rare but clean decay $h \rightarrow \gamma \gamma$ and the high branching ratio decay $h \rightarrow b \bar{b}$ may provide the best opportunity to observe this mode. In this paper, we will therefore explore the ability of the LHC to discover stoponium through $p p \rightarrow \eta_{\tilde{t}} \rightarrow h h \rightarrow \gamma \gamma b \bar{b}$. This could either be an alternative discovery mode, or perhaps a confirmation of a discovery of stoponium in the $\eta_{\tilde{t}} \rightarrow \gamma \gamma$ or $\eta_{\tilde{t}} \rightarrow Z Z$ modes or of open stop pair production.

The stoponium state is produced through gluon fusion, as the near-threshold limit of open stop production. The production cross-section was computed through next-to-leading order (NLO) in ref. [9] in terms of the stoponium wavefunction at the origin. A resummed nextto-next-to-leading logarithm (NNLL) calculation is provided in [11]; the effects of threshold resummation were found to be small. When needed, we will use the results of [9] for convenience. The remaining uncertainties may well be dominated by the imperfect knowledge of the stoponium wavefunctions and production of the excited states. We note in particular that ref. [9] chose to include only the 1 s and 2 s stoponium states in the production crosssection. Although these give most of the production cross-section, there could be additional rate contributions coming from production of higher excited states, if those decay to the $s$-wave states before decaying by annihilation.

More generally, the same signatures used to search for stoponium will apply to any narrow scalar di-Higgs resonance, including the heavier neutral Higgs scalar boson of the Minimal Supersymmetric Standard Model (MSSM), where there is sensitivity especially if tan $\beta$ is not too large [19-21], as well as other extensions of the Standard Model Higgs sector [22 24]. The paper [24] contains a study similar to the present one, but with somewhat different motivations and procedures. A recent search by CMS [25] looks for $p p \rightarrow H \rightarrow h h$, and sets 
95\% confidence level limits of order $5 \mathrm{pb}$ on the production cross-section for $H$ masses below $360 \mathrm{GeV}$, but using channels other than $b b \gamma \gamma$. In another study by ATLAS [26] it is shown that a good sensitivity can be achieved for $m_{H} \geq 600$ while looking at resonances decaying via a pair of Higgs bosons to the $b \bar{b} b \bar{b}$ final state, with $19.5 \mathrm{fb}^{-1}$ of proton-proton collision data at $\sqrt{s}=8 \mathrm{TeV}$. In the rest of this paper, we will use $\eta$ to represent a generic di-Higgs resonance, although stoponium (denoted $\eta_{\tilde{t}}$ ) is our primary motivation. It should be noted that the signature for di-Higgs production is also used, with different kinematic requirements due to the non-resonant production, in order to study the trilinear Higgs self-coupling as a test of the Standard Model, for example see [19, 20, 27 38]. In the present paper this non-resonant Standard Model di-Higgs production is one of the backgrounds.

There are a variety of model-building motivations for light stops. For example, a light stop is required in the MSSM to enable weak-scale baryogenesis [39]. A light stop scenario is also one way of accommodating the observed dark matter relic density [40, 41] through efficient annihilations in the universe, if the lightest supersymmetric particle (LSP) is binolike and $m_{\tilde{t}_{1}}-m_{\tilde{N}_{1}}$ is much smaller than the top quark mass, as the thermal abundance of DM can be reduced in such cases through stop-mediated neutralino annihilations and/or stop co-annihilations [42 45]. The mass difference between the lighter stop and the LSP must be small enough to forbid flavor-preserving two-body decays in order to give the observed dark matter abundance. Finally, the naturalness arguments for "more minimal supersymmetry" [46, 47] generally incorporate light top squarks as a feature.

Recently, constraints on the light stop scenario have become available from ATLAS [4850] and CMS [51, 52], ruling out significant parts of parameter space, including even cases of stops that are nearly degenerate with the LSP. However, there remain several holes in the exclusions, including the cases $m_{\tilde{t}_{1}}-m_{\tilde{N}_{1}} \approx m_{W}+m_{b}$ and $m_{\tilde{t}_{1}}-m_{\tilde{N}_{1}} \approx m_{t}$. Projected constraints by theorists reinterpreting other ATLAS and CMS searches claim [53, 54] to fill in these holes up to about $m_{\tilde{t}_{1}} \approx 250 \mathrm{GeV}$ (so $m_{\eta_{\tilde{t}}} \approx 500 \mathrm{GeV}$ ), even using less than the full data sets of LHC Run 1. However, we prefer to take these exclusion claims as preliminary until and unless they are confirmed by the experimental collaborations. Furthermore, if the stop decays as $\tilde{t}_{1} \rightarrow j j$ through R-parity violation, where $j$ represents a light quark jet, then there are no exclusions at all [55, 56] at present. In this case, it may be that stoponium will be a competitive way to set model-independent limits on light stops for some time. We will consider stoponium masses down to $275 \mathrm{GeV}$, corresponding to top-squark masses down to about $138 \mathrm{GeV}$, so that $\eta_{\tilde{t}} \rightarrow h h$ is kinematically allowed.

\section{EVENT GENERATION AND SIMULATION}

We used Madgraph 5 [57] to generate events simulating $\eta$ production and decay, $p p \rightarrow$ $\eta \rightarrow h h$, in proton-proton collisions at $\sqrt{s}=14 \mathrm{TeV}$. We used the model HEFT, an extension of the tree-level Standard Model to include an additional scalar, which we interpreted as $\eta$, and effective couplings $g g \eta, g g h$, and $\gamma \gamma h$. We modified HEFT to also include a small $\eta h h$ coupling to allow the decay of interest, which was then forced at the level of event generation. The production cross-section for $p p \rightarrow \eta \rightarrow h h$ is taken as an input parameter, in order to 
maximize the generality of the results. We set the Standard Model Higgs boson mass to be $m_{h}=126 \mathrm{GeV}$, and used branching ratios $\operatorname{BR}(h \rightarrow b \bar{b})=0.57$ and $\operatorname{BR}(h \rightarrow \gamma \gamma)=0.0022$.

In order to improve the statistics, we generated signal events in which one of the $h$ was forced to decay to $b \bar{b}$ and the other to $\gamma \gamma$, and then normalized the resulting event sample according to the branching ratios and the assumed $p p \rightarrow \eta \rightarrow h h$ production rate just mentioned. We generated 100,000 events for each of $m_{\eta}=275,300,325,350,375,400,425$, $450,475,500,525,550,575,600,650,700,800,900$, and $1000 \mathrm{GeV}$ in this way. All the signal samples as well as the background samples mentioned below were generated using Madgraph 5 and showered with Pythia 6 [58].

The possible backgrounds include non-resonant $\gamma \gamma b \bar{b}$ production, as well as $\gamma \gamma c \bar{c}$ and $\gamma \gamma j(b / \bar{b})$ and $\gamma \gamma j(c / \bar{c}$ ) and $\gamma \gamma j j$ (where $j=g, u, d, s, \bar{u}, \bar{d}, \bar{s}$ ), and $\gamma \gamma t \bar{t}$ and $\gamma \gamma Z$ and $t \bar{t} h$ and $Z h$ and $b \bar{b} h$ and $h h$. Production of the $h h$ background includes a triangular and a box diagram, but the effective coupling for the latter is not included in the version of HEFT we used. We therefore normalized the cross-section for the $h$ h background to be $40.2 \mathrm{fb}$, from [24]. In the LHC detectors, electrons are sometimes misidentified as photons. We therefore included backgrounds from the processes $t \bar{t}$ (with two electrons faking photons) and $t \bar{t} \gamma$ (with one electron faking a photon). Here we used a probability of 0.0181 for each electron to fake a photon [59]. We did not include a possible 4-jet background ( $j j j j)$ because the efficiencies for two jets to faking photons is very low, and the result must also have two light-flavor jets mis-tagged as $b$-jets with a rate of order $10^{-6}$, and this background tends to be distributed at low photon $p_{T}$ and invariant masses. We did include backgrounds of the form $j \gamma b \bar{b}$, where one jet fakes a photon. Here, we used probabilities 1/20100 for a gluon jet and $1 / 1680$ for a quark jet to fake a photon [60].

In order to obtain good statistics, we found it useful to put a generator-level cut on the minimum and maximum invariant mass of the diphoton pair $\left(106<M_{\gamma \gamma}<146\right)$ in the backgrounds listed above that explicitly include $\gamma \gamma$, because a tighter cut will be imposed at the analysis level anyway. For the $t \bar{t} h$ and $Z h$ and $b \bar{b} h$ backgrounds, we forced $h$ to decay to two photons, and for the $h h$ background we forced one $h$ to decay to $\gamma \gamma$ and the other to decay to $b \bar{b}$, as for the signal. The event samples were normalized accordingly.

For the detector simulation we used Delphes 3 [61]. We chose a conservative $b$-tagging efficiency for $b$-jets of 0.6. The efficiency of mistagging a charm as a $b$-jet was taken to be 0.1 , while for jets initiated by gluons and $u, d, s$ quarks the $b$-jet mistagging efficiency was chosen to be 0.001 .

\section{EVENT SELECTION}

In the analysis, we first selected events with exactly two $b$-tagged jets and two photons. The leading and sub-leading (in transverse momentum, $p_{T}$ ) photon and $b$-jet are denoted $\gamma_{1}, \gamma_{2}$ and $b_{1}, b_{2}$, respectively. We then applied cuts on the $p_{T}$, the pseudo-rapidity $\eta$ and $\left.\Delta R \equiv \sqrt{(\Delta \eta)^{2}+(\Delta \phi)^{2}}\right)$ as follows, referred to below as event selection $\mathbf{S} 1$ :

- $p_{T}\left(b_{1}, b_{2}\right)>(40,30) \mathrm{GeV}$

- $p_{T}\left(\gamma_{1}, \gamma_{2}\right)>(35,25) \mathrm{GeV}$ 
- $\left|\eta\left(b_{1}, b_{2}\right)\right|<2.7$

- $\left|\eta\left(\gamma_{1}, \gamma_{2}\right)\right|<2.5$

- $\Delta R_{i j}>0.5$, for $i, j=b_{1}, b_{2}, \gamma_{1}, \gamma_{2}$

The cuts on $b \bar{b}$ invariant mass, $p_{T}$ and $\Delta R$ has been chosen to retain most of the signal while reducing some major sources of background. In particular, we found that reducing the $\Delta R$ cuts to 0.4 does not increase the signal acceptance by a significant amount. We performed the whole analysis with various other choices of leading and sub-leading $b$-jet $p_{T}$ 's and found that other choices do not provide for a significantly better retention of signal over background.

Given the kinematics of the signal we are interested in, we then applied cuts on the invariant masses of the $\gamma \gamma$ pair, the $b b$ pair, and on the 4-body $\gamma \gamma b b$ system. For the last cut, we found that it is better to define a modified invariant mass $M_{X}$, according to

$$
M_{X} \equiv M_{b b \gamma \gamma}-M_{b b}+m_{h},
$$

where $m_{h}=126 \mathrm{GeV}$ is the fixed, known Higgs mass. By subtracting off $M_{b b}$ and adding in the true Higgs mass, one tends to mitigate the effects of $b$-jet momentum mismeasurements. The distribution of $M_{X}$ has a sharper peak, and is concentrated closer to $m_{\eta}$, than the distribution of $M_{b b \gamma \gamma}$. The sequence of event selection cuts we used is:

S2: As in S1, with $\left|M_{\gamma \gamma}-m_{h}\right|<6 \mathrm{GeV}$,

S3: As in S2, with $\left|M_{b b}-m_{h}\right|<30 \mathrm{GeV}$,

S4: As in S3, with $\left|M_{X}-m_{\eta}\right|<0.07 m_{\eta}$, where $m_{\eta}$ is the position of the putative peak.

The widths of the $M_{\gamma \gamma}$ and $M_{b b}$ cuts are somewhat larger than the resolutions of a sample of single Higgs boson production, reflecting the performance we observed using Delphes when the Higgs bosons originate from heavy $\eta$ decays. Somewhat narrower (wider) windows could perhaps be used for smaller (larger) $m_{\eta}$, although we did not attempt to optimize this, since the optimization is likely to be quite different in real data than in our simulations. The advantage of using $M_{X}$ rather than the usual 4-body invariant mass $M_{b b \gamma \gamma}$ is illustrated in Figure 3.1 for signal events that pass the $\mathbf{S} 3$ selection cuts, for $m_{\eta}=275 \mathrm{GeV}$ and for $m_{\eta}=500 \mathrm{GeV}$. The distributions of $M_{X}$ as defined in eq. (3.1), for various different masses $m_{\eta}$ are shown in Figure 3.2, again after the S3 selection cuts. It can be seen that the $M_{X}$ distributions are peaked near the correct $\eta$ mass, and get wider as $m_{\eta}$ increases. For the larger values of $m_{\eta}$, especially above about $700 \mathrm{GeV}$, the maximum of the $M_{X}$ distribution occurs somewhat above the true mass, but with a much fatter tail below than above. This is an effect that can be corrected for by the experimental collaborations in real data, and in our simulation most events are still within about $\pm 7 \%$ of the true value. Here, we expect that in practice a comparison between Monte Carlo simulations and an observed distribution will allow a hypothesis value of $m_{\eta}$ to be obtained in cases where a peak is present and large enough to possibly allow a 5 -sigma discovery claim. Given the luminosity requirements for a 

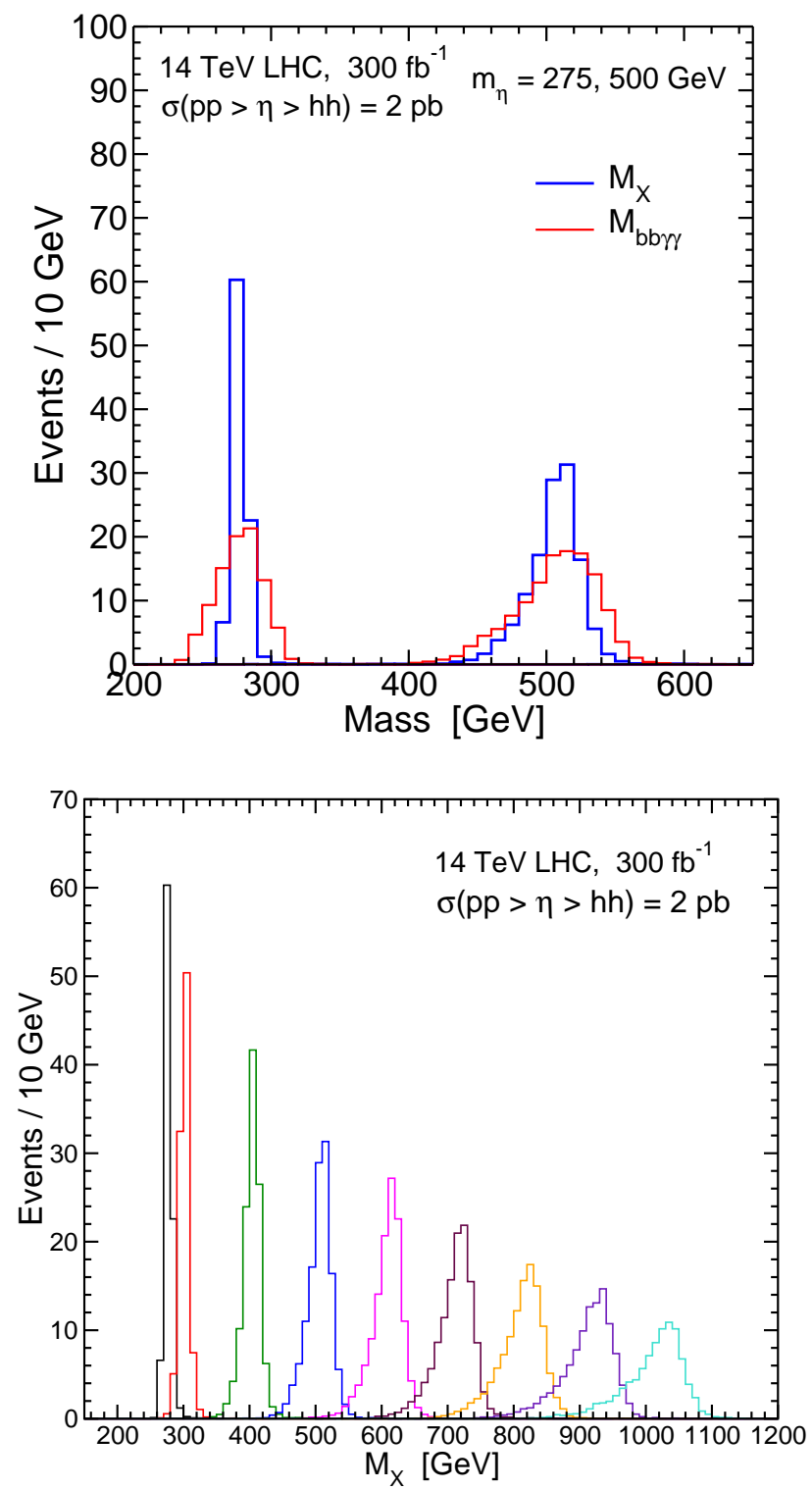

FIG. 3.1: Distributions of $M_{b b \gamma \gamma}$ and $M_{X}$ as defined in eq. (3.1), for input masses $m_{\eta}=$ $275 \mathrm{GeV}$ and $500 \mathrm{GeV}$. Both distributions are based on 100,000 signal events $p p \rightarrow \eta \rightarrow$ $h h$ with one $h$ forced to decay to $\gamma \gamma$ and the other to $b \bar{b}$, and with the distributions normalized by assuming $\sigma \times \mathrm{BR}(p p \rightarrow \eta \rightarrow$ $h h)=2 \mathrm{pb}$ and an integrated luminosity of $300 \mathrm{fb}^{-1}$. The events were selected with the S3 cuts.
FIG. 3.2: Distributions of $M_{X}$ for $m_{\eta}=275$, $300,400,500,600,700,800,900$, and 1000 $\mathrm{GeV}$, for signal events, normalized assuming $\sigma \times \mathrm{BR}(p p \rightarrow \eta \rightarrow h h)=2 \mathrm{pb}$ and an integrated luminosity of $300 \mathrm{fb}^{-1}$, with the event selection $\mathbf{S 3}$ cuts imposed.

stoponium discovery, one may also expect that evidence for a stop, either in open production or in $\eta_{\tilde{t}} \rightarrow \gamma \gamma$ or $Z Z$ will have already accrued to allow for at least a rough estimate of the mass.

The fractions of $p p \rightarrow \eta \rightarrow h$ signal events that pass selections S1, S2, S3, and S4 are given in Table 3.1 for various values of $m_{\eta}$. In order to obtain good statistics, the results were obtained for each $m_{\eta}$ by generating 100,000 events $p p \rightarrow \eta \rightarrow h h$ with one $h$ forced to decay to $\gamma \gamma$ and the other forced to decay to $b \bar{b}$, and then normalizing the results using $\mathrm{BR}(h \rightarrow \gamma \gamma)=0.0022$ and $\mathrm{BR}(h \rightarrow b \bar{b})=0.57$. The nominal fraction of $p p \rightarrow \eta \rightarrow h h$ that will yield $b \bar{b} \gamma \gamma$ before imposing any selection cuts and efficiencies is $2(0.0022)(0.57)=0.00253$. After the $\mathbf{S} 4$ selection cuts, the fraction of signal events surviving is of order $2 \times 10^{-4}$, and is largest for $m_{\eta}$ near $500 \mathrm{GeV}$.

The backgrounds simulated and the cross-sections to pass the selections $\mathbf{S 1}, \mathbf{S 2}, \mathbf{S 3}, \mathbf{S 4}$, are shown in Table 3.2 , for the case that $m_{\eta}=275 \mathrm{GeV}$. (Only the $\mathbf{S} 4$ selection depends on 
TABLE 3.1: The fraction of $p p \rightarrow \eta \rightarrow h h$ signal events at $\sqrt{s}=14 \mathrm{TeV}$ that pass selections $\mathbf{S 1}$, S2, S3, and S4. The results were obtained for each $m_{\eta}$ by generating 100,000 events $p p \rightarrow \eta \rightarrow h h$ with one $h$ forced to decay to $\gamma \gamma$ and the other forced to decay to $b \bar{b}$, and then normalizing the results using $\mathrm{BR}(h \rightarrow \gamma \gamma)=0.0022$ and $\mathrm{BR}(h \rightarrow b \bar{b})=0.57$.

\begin{tabular}{|c|c|c|c|c|}
\hline$p p \rightarrow \eta \rightarrow h h$ & \multicolumn{4}{|c|}{ fraction $\times 10^{4}$} \\
\hline$m_{\eta}(\mathrm{GeV})$ & S1 & S2 & S3 & S4 \\
\hline 275 & 1.88 & 1.80 & 1.52 & 1.51 \\
300 & 2.06 & 1.97 & 1.63 & 1.59 \\
325 & 2.26 & 2.13 & 1.72 & 1.67 \\
350 & 2.43 & 2.23 & 1.79 & 1.72 \\
375 & 2.55 & 2.30 & 1.84 & 1.76 \\
400 & 2.81 & 2.48 & 1.96 & 1.86 \\
425 & 2.91 & 2.49 & 1.98 & 1.87 \\
450 & 3.04 & 2.52 & 2.01 & 1.88 \\
475 & 3.20 & 2.60 & 2.08 & 1.95 \\
500 & 3.29 & 2.63 & 2.11 & 1.95 \\
525 & 3.36 & 2.57 & 2.08 & 1.92 \\
550 & 3.49 & 2.60 & 2.10 & 1.94 \\
575 & 3.47 & 2.53 & 2.05 & 1.88 \\
600 & 3.63 & 2.59 & 2.12 & 1.94 \\
650 & 3.78 & 2.53 & 2.07 & 1.89 \\
700 & 3.95 & 2.52 & 2.09 & 1.90 \\
800 & 4.02 & 2.32 & 1.95 & 1.75 \\
900 & 3.94 & 2.14 & 1.82 & 1.63 \\
1000 & 3.51 & 1.84 & 1.54 & 1.36 \\
\hline
\end{tabular}

the choice of $m_{\eta}$.) In Figure 3.3. we show for $m_{\eta}=275 \mathrm{GeV}$ the $M_{b b}$ distributions for the signal and the background after applying the selections $\mathbf{S 2}$, and again after including the S4 cut on $M_{X}$. The latter cut is seen to strongly reduce the background while keeping most of the signal. In Figure 3.4 we show the $M_{X}$ distributions for the total background and for the signal, assuming $\sigma(p p \rightarrow \eta \rightarrow h h)=2 \mathrm{pb}$, for two choices $m_{\eta}=275$ and $500 \mathrm{GeV}$. The left panel shows the $M_{X}$ distributions after the event selections $\mathbf{S 2}$, and the right panel after including the $\mathbf{S 3}$ selection cut on $M_{b b}$, which clearly helps to give a good discrimination against total background. These distributions are again shown weighted according to 300 $\mathrm{fb}^{-1}$ integrated luminosity. Because the event selection $\mathbf{S} \mathbf{4}$ cut depends on the $m_{\eta}$ of the putative peak, the background drops significantly with higher masses. This is shown in Table 3.3 for $m_{\eta}=300,400,500,600,700,800,900,1000 \mathrm{GeV}$. Note that for smaller $m_{\eta}$, the backgrounds are largest for $\gamma \gamma b \bar{b}$ and $\gamma \gamma j(b / \bar{b})$ and $j \gamma b \bar{b}$, but for higher $m_{\eta}$ we find that the largest background is $\gamma \gamma j j$ for $j=g, u, d, s, \bar{u}, \bar{d}, \bar{s}$. Clearly these results will be dependent on the ability of the detector analyses to minimize mis-tags of gluon and light quark jets as $b$-jets and photons. 
TABLE 3.2: Significant background cross-sections after event selections S1, S2, S3 and S4, for $m_{\eta}=275 \mathrm{GeV}$. The number of events generated, $N_{\text {gen }}$, is also given. In order to improve statistics, the first seven backgrounds with $\gamma \gamma$ were generated with a cut $\left|M_{\gamma \gamma}-m_{h}\right|<20 \mathrm{GeV}$, while the next four backgrounds were generated with $h \rightarrow \gamma \gamma$ forced, and the $h h$ background was generated with one $h$ forced to decay to $\gamma \gamma$ and the other to $b \bar{b}$.

\begin{tabular}{|c|c|c|c|c|c|}
\hline Background & \multirow{2}{*}{$N_{\text {gen }}$} & \multicolumn{4}{|c|}{$\sigma_{\text {pass }}(\mathrm{fb})$} \\
\cline { 3 - 6 } & & $\mathrm{S} 1$ & $\mathrm{~S} 2$ & $\mathrm{S3}$ & $\mathrm{S} 4$ \\
\hline$p p \rightarrow \gamma \gamma b \bar{b}$ & 200000 & 0.944 & 0.284 & 0.0861 & 0.0329 \\
$p p \rightarrow \gamma \gamma c \bar{c}$ & 440000 & 0.303 & 0.0912 & 0.0301 & 0.0131 \\
$p p \rightarrow \gamma \gamma t \bar{t}$ & 200000 & 0.119 & 0.0640 & 0.0176 & 0.00449 \\
$p p \rightarrow \gamma \gamma j(b / \bar{b})$ & 200000 & 0.764 & 0.233 & 0.0818 & 0.0217 \\
$p p \rightarrow \gamma \gamma j(c / \bar{c})$ & 600000 & 0.369 & 0.114 & 0.0337 & 0.0078 \\
$p p \rightarrow \gamma \gamma j j$ & 1200000 & 0.540 & 0.186 & 0.0723 & 0.0723 \\
$p p \rightarrow \gamma \gamma Z$ & 200000 & 0.0462 & 0.0172 & 0.00220 & 0.00052 \\
$p p \rightarrow t \bar{t} h$ & 100000 & 0.0733 & 0.0631 & 0.0171 & 0.00413 \\
$p p \rightarrow Z h$ & 100000 & 0.00919 & 0.00792 & 0.00329 & 0.00066 \\
$p p \rightarrow b \bar{b} h$ & 100000 & 0.0113 & 0.00992 & 0.00251 & 0.00052 \\
$p p \rightarrow h h$ & 100000 & 0.00927 & 0.00838 & 0.00682 & 0.00212 \\
$p p \rightarrow t t$ & 500000 & 0.108 & 0.00748 & 0.00216 & 0.00090 \\
$p p \rightarrow \gamma t t$ & 500000 & 0.157 & 0.00992 & 0.00267 & 0.00086 \\
$p p \rightarrow g \gamma b \bar{b}$ & 500000 & 0.3522 & 0.0314 & 0.0113 & 0.00411 \\
$p p \rightarrow(q / \bar{q}) \gamma b \bar{b}$ & 500000 & 3.568 & 0.253 & 0.0763 & 0.0173 \\
\hline Total & & 7.374 & 1.379 & 0.446 & 0.118 \\
\hline
\end{tabular}
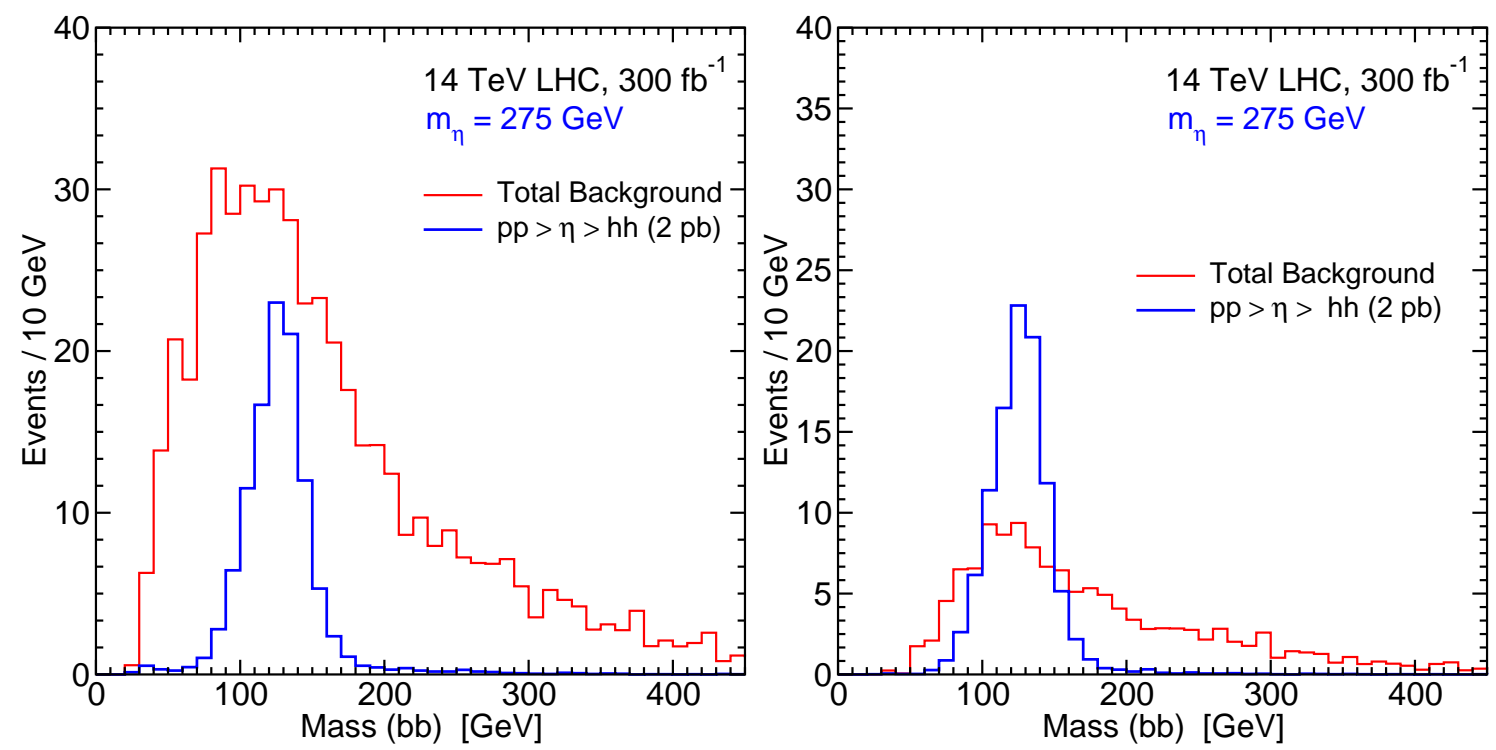

FIG. 3.3: The signal and total background distributions of $M_{b b}$, after applying the S2 cuts (left panel) and after including in addition the $\mathbf{S} 4$ cut $\left|M_{X}-m_{\eta}\right|<0.07 m_{\eta}$ (right panel), for $m_{\eta}=275$ $\mathrm{GeV}$. The normalizations assume $300 \mathrm{fb}^{-1}$ with $\sigma(p p \rightarrow \eta \rightarrow h h)=2 \mathrm{pb}$. 

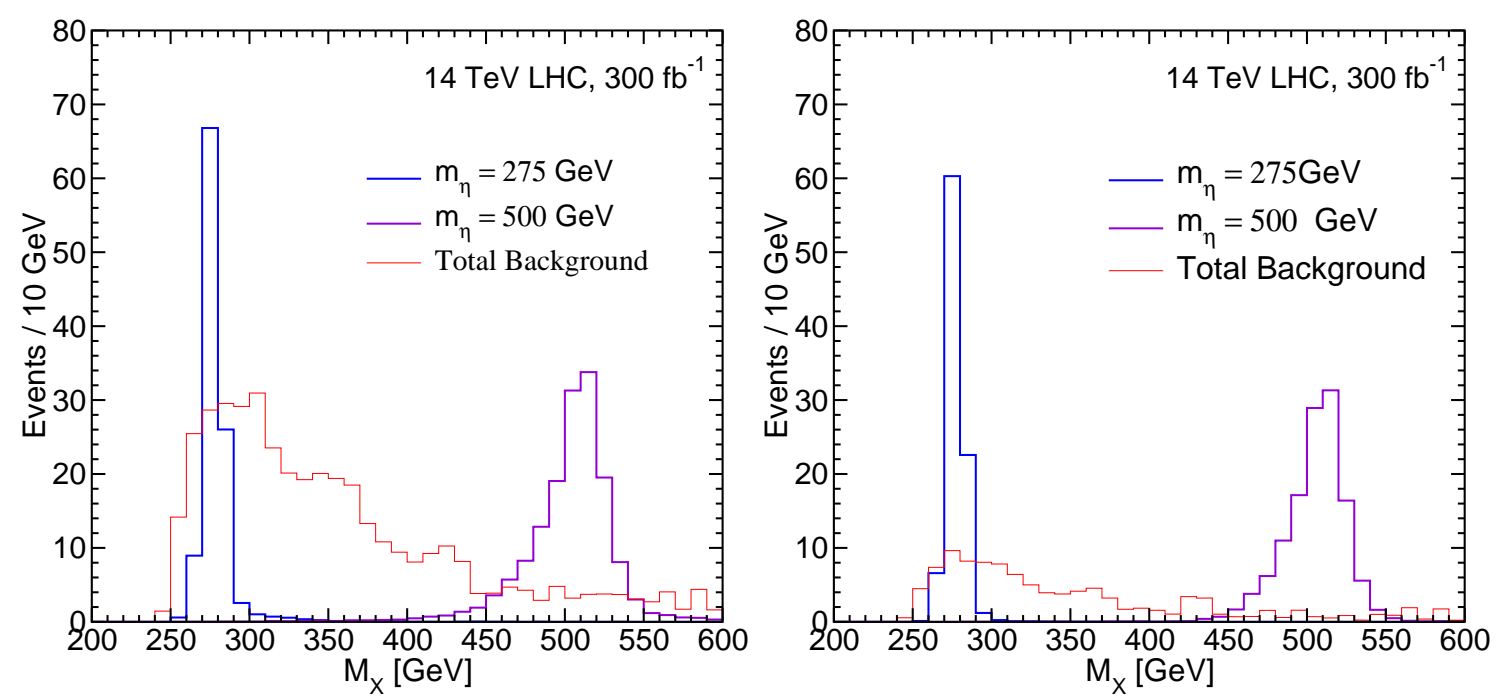

FIG. 3.4: The $M_{X}$ distributions of the total background and the signal are shown after event selections S2 (left panel) and after S3 (right panel). For the signal, the distributions are shown for $m_{\eta}=275 \mathrm{GeV}$ and $500 \mathrm{GeV}$, with $\sigma(p p \rightarrow \eta \rightarrow h h)=2 \mathrm{pb}$ in both cases. The integrated luminosity is taken to be $300 \mathrm{fb}^{-1}$.

TABLE 3.3: Background cross sections in fb after selections $\mathbf{S} 4$, for $m_{\eta}=300,400,500,600,700$, 800, 900, $1000 \mathrm{GeV}$. Cases where no events passed the $\mathbf{S} 4$ selections are listed with $\leq$ and the 1-event cross-section of our sample. For these and other values of $m_{\eta}$, the total backgrounds after the $\mathbf{S} \mathbf{4}$ cuts are shown in Figure 3.5 below.

\begin{tabular}{|c|c|c|c|c|c|c|c|c|}
\hline \multirow{2}{*}{ Background } & \multicolumn{7}{|c|}{$\sigma_{\text {pass }}(\mathrm{fb})$ for various $m_{\eta}$ in $\mathrm{GeV}$} \\
\cline { 2 - 8 } & 300 & 400 & 500 & 600 & 700 & 800 & 900 & 1000 \\
\hline$\gamma \gamma b \bar{b}$ & 0.0291 & 0.00797 & 0.00286 & 0.00082 & 0.00061 & 0.00020 & 0.00041 & 0.00010 \\
$\gamma \gamma c \bar{c}$ & 0.00921 & 0.00146 & 0.00048 & 0.00048 & $\leq 0.00048$ & $\leq 0.00048$ & $\leq 0.00048$ & $\leq 0.00048$ \\
$\gamma \gamma t \bar{t}$ & 0.00497 & 0.00253 & 0.00104 & 0.00045 & 0.00016 & 0.00010 & 0.00003 & 0.00001 \\
$\gamma \gamma j(b / \bar{b})$ & 0.0199 & 0.00938 & 0.00563 & 0.00338 & 0.00525 & 0.00263 & 0.00075 & 0.00037 \\
$\gamma \gamma j(c / \bar{c})$ & 0.01037 & 0.00648 & 0.00389 & 0.00130 & $\leq 0.00130$ & $\leq 0.00130$ & $\leq 0.00130$ & $\leq 0.00130$ \\
$\gamma \gamma j j$ & 0.01446 & 0.00482 & 0.00482 & 0.0121 & $\leq 0.00241$ & 0.00241 & 0.00241 & 0.00482 \\
$\gamma \gamma Z$ & 0.00036 & 0.00040 & 0.00016 & 0.00012 & 0.00008 & 0.00012 & $\leq 0.00004$ & $\leq 0.00004$ \\
$t \bar{t} h$ & 0.00483 & 0.00255 & 0.00088 & 0.00045 & 0.00024 & 0.00006 & 0.00005 & 0.00002 \\
$Z h$ & 0.00066 & 0.00055 & 0.00033 & 0.00018 & 0.00011 & 0.00006 & 0.00002 & 0.00001 \\
$b \bar{b} h$ & 0.00050 & 0.00037 & 0.00023 & 0.00013 & 0.00010 & 0.00007 & 0.00003 & 0.00004 \\
$h h$ & 0.00208 & 0.00080 & 0.00032 & 0.00015 & 0.00005 & 0.00003 & 0.00002 & 0.000004 \\
$t t$ & 0.00091 & 0.00011 & 0.00002 & $\leq 3 \times 10^{-6}$ & $\leq 3 \times 10^{-6}$ & $\leq 3 \times 10^{-6}$ & $\leq 3 \times 10^{-6}$ & $\leq 3 \times 10^{-6}$ \\
$\gamma t t$ & 0.00090 & 0.00028 & 0.00005 & 0.000025 & 0.000035 & 0.000015 & $\leq 5 \times 10^{-6}$ & $\leq 5 \times 10^{-6}$ \\
$g \gamma b \bar{b}$ & 0.00412 & 0.00091 & 0.00030 & 0.00011 & 0.00004 & $\leq 0.00004$ & $\leq 0.00004$ & $\leq 0.00004$ \\
$(q / \bar{q}) \gamma b \bar{b}$ & 0.0187 & 0.0101 & 0.00662 & 0.00576 & 0.00201 & 0.00115 & 0.00058 & 0.00029 \\
\hline Total & 0.1213 & 0.0487 & 0.0276 & 0.0254 & 0.0105 & 0.0087 & 0.0062 & 0.0075 \\
\hline
\end{tabular}




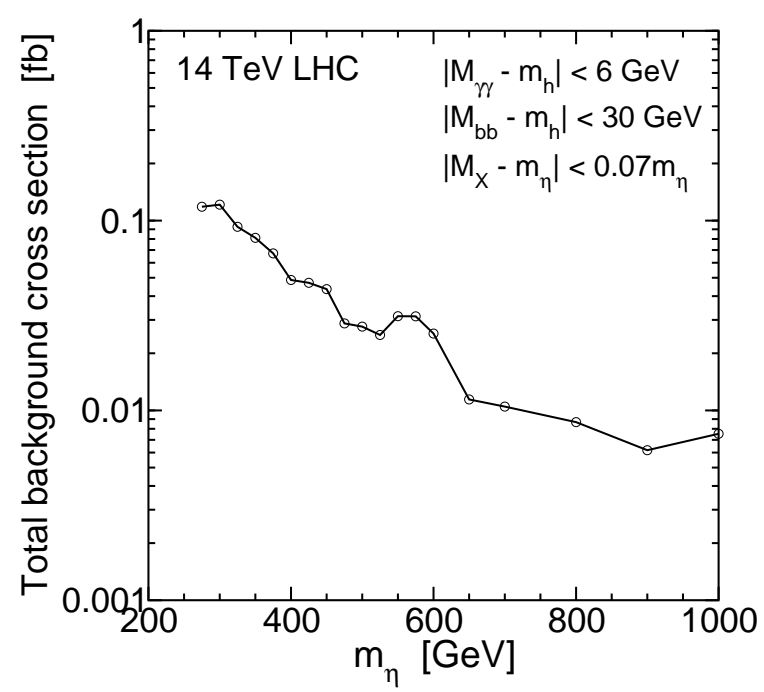

FIG. 3.5: Total background cross section passing all cuts for event selection $\mathbf{S 4}$, as a function of $m_{\eta}$, which enters into the $M_{X}$ cut.

The results for the total background cross-sections passing events selection $\mathbf{S 4}$, as a function of $m_{\eta}$, are plotted in Figure 3.5.

\section{DISCOVERY PROSPECT PROJECTIONS FOR THE 14 TEV LHC}

In actual experimental data, the appearance of a peak in the $M_{X}$ distribution would allow a discovery if it is large enough. The background levels should be determined with some accuracy from data, due to the presence of several sideband control regions. These include events with $M_{\gamma \gamma}$ outside of the window specified in the $\mathbf{S} 2$ cut, events with $M_{b b}$ outside of the window specified in the S3 cut, and events with $M_{X}$ outside of the window specified in the $\mathbf{S} \mathbf{4}$ cut. We therefore assume that the determination of backgrounds for the search will be mostly statistical, and set a requirement for a 5-sigma observation of the signal by demanding that $S / \sqrt{B}>5$, where $S$ and $B$ are the numbers of signal and background events, respectively, that pass the $\mathbf{S} \mathbf{4}$ selection. While this does not account for the "lookelsewhere" effect, it is likely that because of the large luminosities required, by the time a stoponium discovery search becomes relevant, there will be other evidence either from one or both of the channels $\eta_{\tilde{t}} \rightarrow \gamma \gamma$ or $\eta_{\tilde{t}} \rightarrow Z Z$ or from open stop production, or perhaps from stops obtained from gluino decays. We also require a minimum of $S>10$ signal events for a discovery, which becomes important when the signal and background cross-sections are both low.

In Figure 4.1 we show the cross-section $\sigma(p p \rightarrow \eta \rightarrow h h)$ needed for $S / \sqrt{B}>5$ and $S>10$, as a function of $m_{\eta}$, for various integrated luminosities and $\sqrt{s}=14 \mathrm{TeV}$. We see that with an integrated luminosity of $100 \mathrm{fb}^{-1}$ at the $14 \mathrm{TeV} \mathrm{LHC}$, one should be able to discover (or, with the look-elsewhere effect, provide strong evidence for) the resonant process $p p \rightarrow \eta \rightarrow h h$, provided the cross-section exceeds $500 \mathrm{fb}$ to $1.2 \mathrm{pb}$, depending on the mass. Put another way, a di-Higgs resonance with a cross-section for $p p \rightarrow \eta \rightarrow h h$ of $1.2 \mathrm{pb}$ can be easily discovered with less than $100 \mathrm{fb}^{-1}$ of integrated luminosity, independent of its mass as long as it is larger than about $275 \mathrm{GeV}$. With $300 \mathrm{fb}^{-1}$, it may be possible to discover a di- 

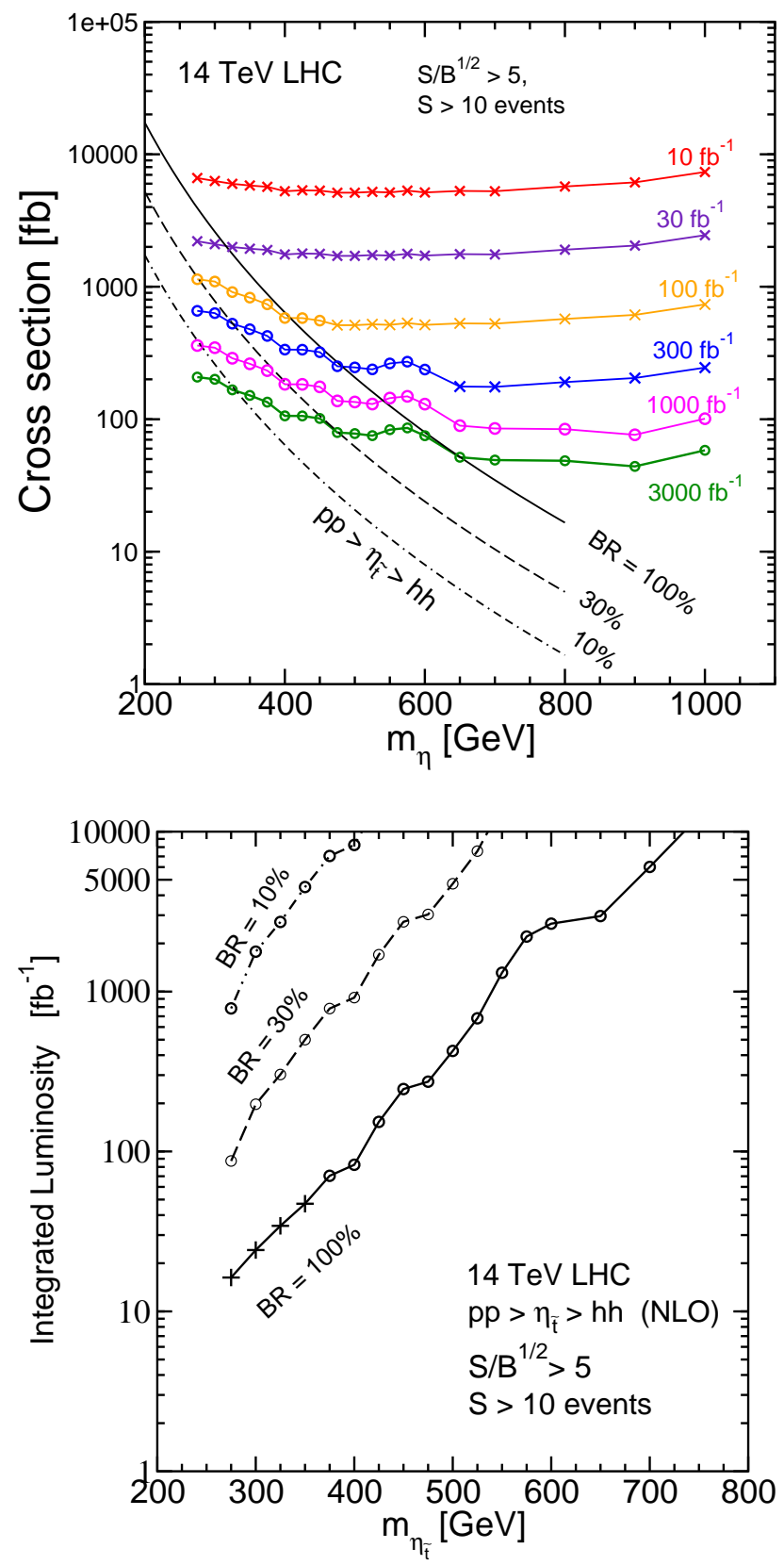

FIG. 4.1: The $\sigma(p p \rightarrow \eta \rightarrow h h)$ cross sections needed for an expected $S / \sqrt{B}>5$, $S>10$ event discovery as a function of $m_{\eta}$, for integrated luminosities 10, 30, 100, 300,1000 , and $3000 \mathrm{fb}^{-1}$ in $p p$ collisions at $\sqrt{s}=14 \mathrm{TeV}$. The points marked with a circle are those that have an expected $S / \sqrt{B}=5$ with $S>10$ events, while those marked with an X have an expected $S=10$ events with $S / \sqrt{B}>5$. Also shown are the predicted cross-sections for stoponium production, $\sigma\left(p p \rightarrow \eta_{\tilde{t}} \rightarrow h h\right)$, based on ref. [9] for NLO $\sigma\left(p p \rightarrow \eta_{\tilde{t}}\right)$ and with assumed $\operatorname{BR}\left(\eta_{\tilde{t}} \rightarrow h h\right)=100 \%, 30 \%, 10 \%$.

FIG. 4.2: Total integrated luminosity needed for an expected $S / \sqrt{B}>5$ and $S>10$ events, as a function of $m_{\eta_{\tilde{t}}}$, for $p p \rightarrow \eta_{\tilde{t}} \rightarrow$ $h h$ at $\sqrt{s}=14 \mathrm{TeV}$, taking the NLO crosssection for $p p \rightarrow \eta_{\tilde{t}}$ from ref. [9] and assuming $100 \%, 30 \%$, and $10 \%$ branching ratios for $\eta_{\tilde{t}} \rightarrow h h$. The points marked with a circle have an expected $S / \sqrt{B}=5$ and $S>10$ events, while those marked by a + symbol have an expected $S=10$ events and $S / \sqrt{B}>5$.

Higgs resonance with a cross-section as low as $175-250 \mathrm{fb}$, if its mass is in the $600-1000 \mathrm{GeV}$ range, although this is limited by statistics. However, for the specific case of stoponium, the expected cross-sections fall very steeply with mass. For comparison, also shown in Figure 4.1 are the predicted cross-sections for stoponium production, $\sigma\left(p p \rightarrow \eta_{\tilde{t}} \rightarrow h h\right)$, based on ref. [9] for $\sigma\left(p p \rightarrow \eta_{\tilde{t}}\right)$ and with assumed $\operatorname{BR}\left(\eta_{\tilde{t}} \rightarrow h h\right)=100 \%, 30 \%$, and $10 \%$, as indicated. Figure 4.2 shows the integrated luminosity required for discovery of stoponium as a function of $m_{\eta_{\tilde{t}}}$, for $100 \%, 30 \%$, and $10 \%$ branching ratios of $\eta_{\tilde{t}}$. With as little as $17 \mathrm{fb}^{-1}$ at $\sqrt{s}=14$ $\mathrm{TeV}$, the LHC could be able to discover the di-Higgs decay of stoponium with $m_{\eta_{\tilde{t}}}=275$ $\mathrm{GeV}$, if the branching ratio for $\eta_{\eta_{\tilde{t}}} \rightarrow h h$ is close to $100 \%$. However, even in this optimistic branching ratio case, the discovery potential with $300 \mathrm{fb}^{-1}$ runs out for stoponium masses heavier than about $500 \mathrm{GeV}$, corresponding to a $250 \mathrm{GeV}$ top squark. For lower branching 
ratios, the required integrated luminosity is clearly much higher.

\section{OUTLOOK}

In this paper we have examined the prospects of detecting stoponium and other di-Higgs resonances in the $b \bar{b} \gamma \gamma$ channel at the LHC with $\sqrt{s}=14 \mathrm{TeV}$. Our results outlined in the previous section can be compared with the heavy Higgs search projections using the same final state made in ref. [24], which we became aware of while the present work was in progress. Ref. [24] used a somewhat different set of analysis parameters, including a higher $b$-tagging efficiency of 0.7 compared to our more conservative 0.6 , a significantly smaller $M_{b b}$ window, and various other different choices for cuts. Nevertheless, comparing our results to Table III of ref. 24] for the case of a $300 \mathrm{GeV}$ scalar, we find a quite similar projection for the $S / \sqrt{B}$. Other results in ref. [24] are based on the particular $(\alpha, \beta)$ parameter space of two Higgs doublet models, so that direct comparisons are difficult for other mass cases. Our work is therefore complementary to ref. [24] in the sense that we presented our projections without tying to a specific model for the production cross-section.

In this paper, we did not attempt to make projections for the ability of the LHC to produce 95\% confidence level exclusions for stoponium or other di-Higgs resonances, which will be appropriate in the case of an absence of any significant candidate peaks in the $b b \gamma \gamma$ invariant mass distribution. To do this will require more sophisticated analyses techniques, rather than just simple cuts. However, clearly the sensitivity of the LHC to making exclusions should be considerably stronger than the discovery projections made here. Besides the $b b \gamma \gamma$ final state looked at here, other channels with higher rates are worthy of consideration [19]-[25]. In any case, it should be clear on general grounds that LHC searches for di-Higgs resonances should be a priority in the future, in order to exploit the Higgs discovery as a possible window to new physics.

Note added: after this paper appeared, the ATLAS collaboration released the results 62] for searches for resonant and non-resonant $h h$ production in the $\gamma \gamma b b$ final state, with $\sqrt{s}=8 \mathrm{TeV}$. The $95 \%$ exclusion on the cross-section at $\sqrt{s}=8 \mathrm{TeV}$ varies from 800 to 3500 $\mathrm{fb}$ when the resonance mass is less than $500 \mathrm{GeV}$, and is weaker than expected for some resonance masses below $350 \mathrm{GeV}$.

Acknowledgments: We thank Jahred Adelman and an anonymous referee for useful comments, and Chul Kim, Ahmad Idilbi, Thomas Mehen, and Yeo Woong Yoon for communications regarding the stoponium production cross-section calculation of ref. [11]. This work was supported in part by the National Science Foundation grant number PHY-1068369.

[1] G. Aad et al. [ATLAS Collaboration], "Observation of a new particle in the search for the Standard Model Higgs boson with the ATLAS detector at the LHC," Phys. Lett. B 716, 1 (2012) [1207.7214], 
[2] [ATLAS Collaboration], "Combined measurements of the mass and signal strength of the Higgs-like boson with the ATLAS detector using up to $25 \mathrm{fb}^{-1}$ of proton-proton collision data," ATLAS-CONF-2013-014, March 6, 2013.

[3] S. Chatrchyan et al. [CMS Collaboration], "Observation of a new boson at a mass of $125 \mathrm{GeV}$ with the CMS experiment at the LHC," Phys. Lett. B 716, 30 (2012) [1207.7235].

[4] [CMS Collaboration], "Combination of standard model Higgs boson searches and measurements of the properties of the new boson with a mass near 125 GeV" CMS-PAS-HIG-12-045, November 16, 2012.

[5] M. Drees and M.M. Nojiri, "A New Signal For Scalar Top Bound State Production," Phys. Rev. Lett. 72, 2324 (1994) [hep-ph/9310209].

[6] M. Drees and M.M. Nojiri, "Production and decay of scalar stoponium bound states," Phys. Rev. D 49, 4595 (1994) hep-ph/9312213].

[7] S.P. Martin, "Diphoton decays of stoponium at the Large Hadron Collider," Phys. Rev. D 77, 075002 (2008) [0801.0237].

[8] S.P. Martin and J.E. Younkin, "Radiative corrections to stoponium annihilation decays," Phys. Rev. D 80, 035026 (2009) [0901.4318].

[9] J.E. Younkin and S.P. Martin, "QCD corrections to stoponium production at hadron colliders," Phys. Rev. D 81, 055006 (2010) [0912.4813].

[10] V. Barger, M. Ishida and W.-Y. Keung, "Searching for Stoponium along with the Higgs boson," Phys. Rev. Lett. 108, 081804 (2012) [1110.2147].

[11] C. Kim, A. Idilbi, T. Mehen and Y. W. Yoon, "Production of Stoponium at the LHC," [1401.1284].

[12] P. Moxhay and R.W. Robinett, "Searching For Scalar Quarkonium At Proton - Anti-Proton Colliders," Phys. Rev. D 32, 300 (1985).

[13] M.J. Herrero, A. Mendez and T.G. Rizzo, "Production of heavy squarkonium at high-energy p p colliders," Phys. Lett. B 200, 205 (1988).

[14] V.D. Barger and W.Y. Keung, "Stoponium decays to Higgs bosons," Phys. Lett. B 211, 355 (1988).

[15] H. Inazawa and T. Morii, " $\overline{\tilde{t}}$ bound state production at multi - TeV hadron colliders," Phys. Rev. Lett. 70, 2992 (1993).

[16] D. S. Gorbunov and V. A. Ilyin, "Stoponium search at photon linear collider," JHEP 0011, 011 (2000) hep-ph/0004092.

[17] D. S. Gorbunov, V. A. Ilyin and V. I. Telnov, "TESLA: Potentials of gamma gamma and e+ e- options in stoponium searches," Nucl. Instrum. Meth. A 472, 171 (2001) hep-ph/0012175.

[18] N. Fabiano, "Estimates of threshold cross-section for stoponium production at e+ e- colliders," Eur. Phys. J. C 19, 547 (2001) hep-ph/0103006.

[19] T. Plehn, M. Spira and P. M. Zerwas, "Pair production of neutral Higgs particles in gluon-gluon collisions," Nucl. Phys. B 479, 46 (1996) [Erratum-ibid. B 531, 655 (1998)] [hep-ph/9603205].

[20] U. Baur, T. Plehn and D. L. Rainwater, "Probing the Higgs selfcoupling at hadron colliders using rare decays," Phys. Rev. D 69, 053004 (2004) [hep-ph/0310056].

[21] M. J. Dolan, C. Englert and M. Spannowsky, "New Physics in LHC Higgs boson pair production," Phys. Rev. D 87, no. 5, 055002 (2013) [1210.8166].

[22] J. Liu, X. P. Wang and S. h. Zhu, "Discovering extra Higgs boson via pair production of the SM-like Higgs bosons," [1310.3634].

[23] J. M. No and M. Ramsey-Musolf, "Probing the Higgs Portal at the LHC Through Resonant di-Higgs Production," [1310.6035].

[24] N. Chen, C. Du, Y. Fang and L. C. Lu, "The LHC Searches for The Heavy Higgs via Two B-jets plus Diphoton," [1312.7212].

[25] CMS Collaboration [CMS Collaboration], "Search for extended Higgs sectors in the H to hh 
and $\mathrm{A}$ to $\mathrm{Zh}$ channels in $\operatorname{sqrt}(\mathrm{s})=8 \mathrm{TeV}$ pp collisions with multileptons and photons final states," CMS-PAS-HIG-13-025.

[26] ATLAS collaboration [ATLAS collaboration], "A search for resonant Higgs-pair production in the 4B-jet final state in pp collisions at 8 TeV" ATLAS-CONF-2014-005.

[27] E. W. N. Glover and J. J. van der Bij, "Higgs Boson Pair Production Via Gluon Fusion," Nucl. Phys. B 309, 282 (1988).

[28] S. Dawson, S. Dittmaier and M. Spira, "Neutral Higgs boson pair production at hadron colliders: QCD corrections," Phys. Rev. D 58, 115012 (1998) hep-ph/9805244.

[29] U. Baur, T. Plehn and D. L. Rainwater, "Determining the Higgs boson selfcoupling at hadron colliders," Phys. Rev. D 67, 033003 (2003) hep-ph/0211224.

[30] M. J. Dolan, C. Englert and M. Spannowsky, "Higgs self-coupling measurements at the LHC," JHEP 1210, 112 (2012) [1206.5001].

[31] A. Papaefstathiou, L. L. Yang and J. Zurita, "Higgs boson pair production at the LHC in the $b \bar{b} W^{+} W^{-}$channel," Phys. Rev. D 87, 011301 (2013) [1209.1489].

[32] J. Baglio, A. Djouadi, R. Gröber, M. M. Mühlleitner, J. Quevillon and M. Spira, "The measurement of the Higgs self-coupling at the LHC: theoretical status," JHEP 1304, 151 (2013) [1212.5581].

[33] F. Goertz, A. Papaefstathiou, L. L. Yang and J. Zurita, "Higgs Boson self-coupling measurements using ratios of cross sections," JHEP 1306, 016 (2013) [1301.3492].

[34] D. Y. Shao, C. S. Li, H. T. Li and J. Wang, "Threshold resummation effects in Higgs boson pair production at the LHC," JHEP 1307, 169 (2013) [arXiv:1301.1245 [hep-ph]].

[35] D. de Florian and J. Mazzitelli, "Two-loop virtual corrections to Higgs pair production," Phys. Lett. B 724, 306 (2013) [1305.5206].

[36] A. J. Barr, M. J. Dolan, C. Englert and M. Spannowsky, "Di-Higgs final states augMT2ed selecting $h h$ events at the high luminosity LHC," Phys. Lett. B 728, 308 (2014) [1309.6318].

[37] D. de Florian and J. Mazzitelli, "Higgs Boson Pair Production at Next-to-Next-to-Leading Order in QCD," Phys. Rev. Lett. 111, 201801 (2013) [1309.6594].

[38] M. J. Dolan, C. Englert, N. Greiner and M. Spannowsky, "Further on up the road: $h h j j$ production at the LHC," [1310.1084].

[39] J.R. Espinosa, M. Quiros and F. Zwirner, Phys. Lett. B 307, 106 (1993) hep-ph/9303317], M.S. Carena, M. Quiros and C.E.M. Wagner, Phys. Lett. B 380, 81 (1996) hep-ph/9603420], Nucl. Phys. B 524, 3 (1998) hep-ph/9710401], J.R. Espinosa, Nucl. Phys. B 475, 273 (1996) hep-ph/9604320, D. Bodeker, P. John, M. Laine and M. G. Schmidt, Nucl. Phys. B 497, 387 (1997) |hep-ph/9612364], M.S. Carena, M. Quiros, A. Riotto, I. Vilja and C.E.M. Wagner, Nucl. Phys. B 503, 387 (1997) hep-ph/9702409, J.M. Cline, M. Joyce and K. Kainulainen, Phys. Lett. B 417, 79 (1998) [Erratum-ibid. B 448, 321 (1999)] hep-ph/9708393], JHEP 0007, 018 (2000) hep-ph/0006119, J.M. Cline and G.D. Moore, Phys. Rev. Lett. 81, 3315 (1998) [hep-ph/9806354], M.S. Carena, M. Quiros, M. Seco and C.E.M. Wagner, Nucl. Phys. B 650, 24 (2003) |hep-ph/0208043]. C. Balazs, M.S. Carena and C.E.M. Wagner, Phys. Rev. D 70, 015007 (2004) (hep-ph/0403224]. C. Balazs, M.S. Carena, A. Menon, D.E. Morrissey and C.E.M. Wagner, Phys. Rev. D 71, 075002 (2005) hep-ph/0412264. D. E. Morrissey and M. J. Ramsey-Musolf, New J. Phys. 14, 125003 (2012) [1206.2942].

[40] C. L. Bennett et al. [WMAP Collaboration], "Nine-Year Wilkinson Microwave Anisotropy Probe (WMAP) Observations: Final Maps and Results," Astrophys. J. Suppl. 208, 20 (2013) [1212.5225].

[41] P. A. R. Ade et al. [Planck Collaboration], "Planck 2013 results. XVI. Cosmological parameters," [1303.5076].

[42] C. Boehm, A. Djouadi and M. Drees, "Light scalar top quarks and supersymmetric dark matter," Phys. Rev. D 62, 035012 (2000) hep-ph/9911496;

[43] J.R. Ellis, K.A. Olive and Y. Santoso, "Calculations of neutralino stop coannihilation in the 
CMSSM," Astropart. Phys. 18, 395 (2003) hep-ph/0112113;

[44] S.P. Martin, "Compressed supersymmetry and natural neutralino dark matter from top squark-mediated annihilation to top quarks," Phys. Rev. D 75, 115005 (2007) hep-ph/0703097 [HEP-PH]]; "The top squark-mediated annihilation scenario and direct detection of dark matter in compressed supersymmetry," Phys. Rev. D 76, 095005 (2007) [hep-ph/0707.2812].

[45] A. De Simone, G. F. Giudice and A. Strumia, "Benchmarks for Dark Matter Searches at the LHC," [1402.6287].

[46] A. G. Cohen, D. B. Kaplan and A. E. Nelson, "The More minimal supersymmetric standard model," Phys. Lett. B 388, 588 (1996) [hep-ph/9607394].

[47] M. Papucci, J. T. Ruderman and A. Weiler, "Natural SUSY Endures," JHEP 1209, 035 (2012) [1110.6926].

[48] ATLAS collaboration, "Search for pair-produced top squarks decaying into a charm quark and the lightest neutralinos with $20.3 \mathrm{fb}^{-1}$ of $p p$ collisions at $\sqrt{s}=8 \mathrm{TeV}$ with the ATLAS detector at the LHC," ATLAS-CONF-2013-068.

[49] ATLAS collaboration, "Search for direct top squark pair production in final states with two leptons in $\sqrt{s}=8 \mathrm{TeV}$ pp collisions using $20 \mathrm{fb}^{-1}$ of ATLAS data.," ATLAS-CONF-2013-048.

[50] G. Aad et al. [ATLAS Collaboration], "Search for direct top-squark pair production in final states with two leptons in pp collisions at $\operatorname{sqrt}(\mathrm{s})=8 \mathrm{TeV}$ with the ATLAS detector," [1403.4853].

[51] S. Chatrchyan et al. [CMS Collaboration], "Search for top-squark pair production in the single-lepton final state in pp collisions at $\sqrt{s}=8 \mathrm{TeV}, "$ Eur. Phys. J. C 73, 2677 (2013) [1308.1586].

[52] CMS Collaboration [CMS Collaboration], "Search for top squarks decaying to a charm quark and a neutralino in events with a jet and missing transverse momentum," CMS-PAS-SUS-13009.

[53] K. Krizka, A. Kumar and D. E. Morrissey, "Very Light Scalar Top Quarks at the LHC," Phys. Rev. D 87, no. 9, 095016 (2013) [1212.4856].

[54] A. Delgado, G. F. Giudice, G. Isidori, M. Pierini and A. Strumia, "The light stop window," Eur. Phys. J. C 73, 2370 (2013) [1212.6847].

[55] J. A. Evans and Y. Kats, "LHC Coverage of RPV MSSM with Light Stops," JHEP 1304, 028 (2013) [1209.0764].

[56] Y. Bai, A. Katz and B. Tweedie, "Pulling Out All the Stops: Searching for RPV SUSY with Stop-Jets," JHEP 1401, 040 (2014) [1309.6631].

[57] J. Alwall, M. Herquet, F. Maltoni, O. Mattelaer and T. Stelzer, "MadGraph 5 : Going Beyond," JHEP 1106, 128 (2011) [1106.0522].

[58] T. Sjostrand, S. Mrenna and P. Z. Skands, "PYTHIA 6.4 Physics and Manual," JHEP 0605, 026 (2006) hep-ph/0603175.

[59] [CMS Collaboration], "Search for supersymmetry in events with photons and missing energy," CMS-PAS-SUS-12-018.

[60] [ATLAS Collaboration], "Expected photon performance in the ATLAS experiment," ATLPHYS-PUB-2011-007.

[61] J. de Favereau, C. Delaere, P. Demin, A. Giammanco, V. Lemaitre, A. Mertens and M. Selvaggi, "DELPHES 3, A modular framework for fast simulation of a generic collider experiment," [1307.6346].

[62] G. Aad et al. [ATLAS Collaboration], "Search for Higgs Boson Pair Production in the $\gamma \gamma b \bar{b}$ Final State using $p p$ Collision Data at $\sqrt{s}=8 \mathrm{TeV}$ from the ATLAS Detector," arXiv:1406.5053 [hep-ex]. 